\title{
Permanent Magnet Motor with Reversible Salient Poles and Variable Magnetic Force
}

\author{
Kazuto Sakai $^{* a)}$ Senior Member, Kouki Matsuda* Student Member
}

(Manuscript received Feb. 1, 2016, revised July 28, 2016)

\begin{abstract}
To operate motors at a higher efficiency over a wider range of speeds, we have developed a technique that quickly reverses the salient poles in permanent magnet (PM) motors. With this technique, PM motors can change negative salient poles into positive salient poles by applying the magnetization of the PM. Furthermore, we discuss the combination of reversible salient poles with variable magnetic force to increase the efficiency of the motor under partial load. This paper proposes a novel PM motor that uses our proposed technique to reverse saliency and describes the principle of reversible pole saliency as well as the resulting motor characteristics. When the proposed motor operates with a negative salient pole, a higher torque is produced at low speeds; when the motor reverses to a positive salient pole, higher power and efficiency are produced at high speeds. These results confirm that the proposed motor can reverse the saliency direction of the pole and that this reversibility allows the motor to achieve high torque at low speeds and high power at high speeds. Consequently, the motor can operate over a wide range of speeds.
\end{abstract}

Keywords: permanent magnet motor, salient pole, magnetization, variable speed, high efficiency

\section{Introduction}

In variable-speed drive systems, such as electric vehicles (EVs) and wind generators, motors operate over a wide range of speeds. Permanent magnet (PM) motors, whose speed can be varied over a wide range by the application of novel methods for changing the magnetic flux, have been proposed in previous studies ${ }^{(1)-(11)}$. Weschta proposed a PM motor in which demagnetization of the PM causes the magnetic flux to vary, reducing the induced voltage at high speeds ${ }^{(1)}$. Ostovic proposed the concept of a memory motor that could vary the magnetic flux and the number of poles of a $\mathrm{PM}^{(3)(4)}$. Sakai et al. proposed a motor with variable magnetic force in which the magnetic flux can be modulated by demagnetizing and remagnetizing a $\mathrm{PM}^{(5)-(7)}$. To improve the efficiency of that motor, Sakai et al. also proposed a PM motor that could vary the number of poles ${ }^{(8)(9)}$. Because the performance of this pole-changing depends on speed, variable-speed drive systems (for instance, EVs) are enabled to operate under low power consumption. Also, Kato, Lorenz et al. proposed a variable magnetomotive force-intensified interior PM (IPM) motor ${ }^{(10)}$. As an alternative method, Sakai et al. proposed a reversible salient pole motor in which the d-axis pole could be reversed to the q-axis by magnetizing a $\mathrm{PM}^{(11)-(13)}$.

This paper proposes a novel design for a reversible salientpole permanent magnet (RS-PM) motor, which can change negative salient poles into positive ones by magnetizing the PM. The results of our analysis confirm that the RS-PM motor has both negative and positive saliency and that the poles can be reversed between these directions. In addition, we

a) Correspondence to: Kazuto Sakai. E-mail: k.sakai @ toyo.jp

* Electrical, Electronic and Communications Engineering, Toyo University

2100, Kujirai, Kawagoe, Saitama 350-8585, Japan discuss the combination of reversible saliency with variable magnetic force in a PM to increase motor efficiency under partial loading. The reversible salient pole allows the motor to achieve high torque at low speeds and high power at high speeds while operating efficiently over a wide speed range.

\section{Principles of the RS-PM Motor}

The RS-PM motor is similar in construction to an IPM motor. Figure 1 shows the basic configuration of the proposed RS-PM motor, which can reverse saliency. The rotor has an iron-salient core in which the PMs are embedded. There are two types of PMs: one with a low coercive force (variablemagnetization magnet or VMM) and one with a high coercive force (constant-magnetization magnet or CMM). We refer to the use of negative salient poles as the "NS-mode" and that of positive salient poles as the "PS-mode." In the NS-mode, four pairs of PMs are arranged into a V shape in the rotor core (Fig. 1(a)); each pair of PMs consists of a VMM and a CMM. Both types of magnets in the V-shaped construct

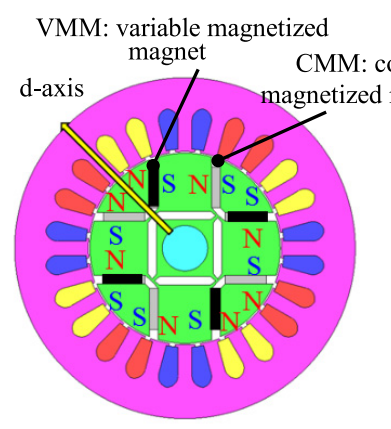

(a) NS-mode (Negative salient pole)

Fig. 1. Configuration of

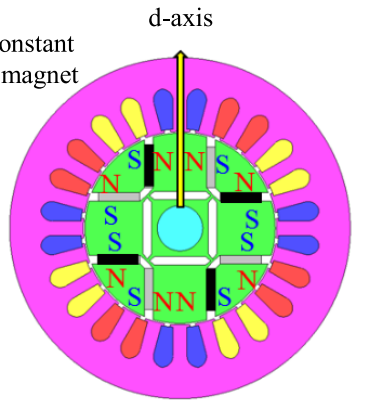

(b) PS-mode (Positive salient pole) 
have the same polarity; thus, the center line of the $\mathrm{V}$ shape is the machine's d-axis. Subsequently, the motor reverses its polarity from negative saliency to (assumed to be) positive saliency, which is the PS-mode. When the negative d-axis pulse current of the armature winding flows, only the VMMs in the rotor are magnetized by the magnetic field of the pulse current. The amplitude of the pulse current ranges from two to ten times that of the rated current while the width is about $10 \mathrm{~ms}$. This implies that the conduction time of the pulse current is about $10 \mathrm{~ms}$, and the VMMs reverse polarity, as shown in Fig. 1(b). As a result, the center line of the $\mathrm{V}$ shape is the machine's q-axis, and that between the two $\mathrm{V}$ shapes is the machine's d-axis. Thus, the d-axis and q-axis exchange positions with one another.

\section{Characteristics of the Reversible Salient Pole}

We discuss the motor's characteristics, including the magnetization of the PM and the motor's performance at variable speed due to reversal of the salient pole using a basic model of the RS-PM motor. Finite-element method (FEM) magneticfield analysis was performed using the FEM software JMAG to verify the proposed RS-PM motor's feasibility and to examine the magnetization of the PM and various motor characteristics caused by having a reversible salient pole.

3.1 Model of the RS-PM Motor We consider how the magnetization of the PM achieves a reversible salient pole, as well as the motor characteristics allowed by changes in saliency. A model of the RS-PM motor for this analysis is shown in Fig. 1. The RS-PM motor includes two types of PMs in its rotor. One has a high magnetic coercive force $(1,000 \mathrm{kA} / \mathrm{m}$ in this analytical model) and is called a constant-magnetization magnet (CMM). The other type has a low coercive force (around $150-160 \mathrm{kA} / \mathrm{m}$ in this model), and is called a variable-magnetization magnet (VMM). The two types of PMs are arranged in a V-shaped pattern in the rotor core. The analytical RS-PM motor has two rotor models with different $\mathrm{V}$-shaped arcs: these arcs have interior angles of $90^{\circ}$ and $100^{\circ}$, as shown in Fig. 2. These values were set based on the literature ${ }^{(11)(12)}$, which implied that they would offer optimal properties in terms of both torque and variable speed, thereby allowing us to study the important characteristics of the motor in detail. Table 1 shows the specifications of the analytical RS-PM motor model.

3.2 Magnetization Characteristics of the Reversible Salient Pole The rotor can reverse the salient pole when the VMMs are magnetized. That is, the motor can change from the NS-mode to the PS-mode and back again. Magnetic field analysis was used to determine whether changes in the magnetization of the VMMs can reverse the polarity. The magnetization was analyzed after the magnetizing current had flowed through the armature winding. The d-axis current corresponds to the magnetizing current in the newly established pole.

We analyzed the magnetization characteristics associated with changing the salient pole from negative to positive and vice versa (or reversing the polarity of the VMMs) by reversing the direction of the field applied to the VMMs. Figures 3 and 4 show the magnetizations of the VMMs and CMMs, respectively, that are associated with changes from the NSmode to the PS-mode in the RS-PM motor, while Figs. 5 and

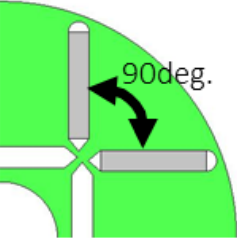

(a) V-shaped arc: $90^{\circ}$

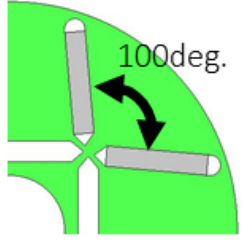

(b) V-shaped arc: $100^{\circ}$
Fig. 2. Rotor with different V-shaped arcs

Table 1. RS-PM motor specifications

\begin{tabular}{cc}
\hline Design item & Setting value \\
\hline Number of pole & 4 \\
\hline Diameter of stator & $120 \mathrm{~mm}$ \\
\hline Diameter of rotor & $67 \mathrm{~mm}$ \\
\hline Core length & $50 \mathrm{~mm}$ \\
\hline Air gap length & $0.5 \mathrm{~mm}$ \\
\hline Number of turns in armature coil (turn/slot) & $3 \mathrm{~mm}$ \\
\hline Rotational speed & 48 turns \\
\hline Rated current & $3000 \mathrm{rpm}$ \\
\hline Current density & $5 \mathrm{Arms}$ \\
\hline Thickness of magnetized magnet & $4 \mathrm{~A} / \mathrm{mm}$ \\
\hline Material of variable magnetized magnet & SmCo (with low \\
\hline Residual flux density in PM & $1.2 \mathrm{~T}$ \\
\hline Coercive force in PM & $150,160 \mathrm{kA} / \mathrm{m}$ \\
\hline Material of constant magnetized magnet & $\mathrm{NdFeB}$ \\
\hline Residual flux density in PM & $1.3 \mathrm{~T}$ \\
\hline Coercive force in PM & $1000 \mathrm{kA} / \mathrm{m}$ \\
\hline
\end{tabular}

6 show the magnetizations of the VMMs and CMMs, respectively, that are associated with changes from the PS-mode to the NS-mode. Here $1 \mathrm{pu}$ is the rated current (current density $=4 \mathrm{~A} / \mathrm{mm}^{2}$ ), which shifts the phase of the current flow of the armature windings to magnetize the VMMs. The results indicate that the phase shift allows the magnetizing current to be smaller if both the d- and q-axes exhibit current flow. As a result, a magnetizing current of 4 pu can magnetize only the VMMs, with the magnetization varying from 100 to $-100 \%$ for polarity reversal. The CMMs are not demagnetized and maintain the state of previous magnetization. We repeated this analysis for the magnetization of the VMMs necessary to switch from the PS-mode to the NS-mode, and the magnetization characteristics associated with applying the reverse field (opposite direction) were obtained. The results indicate that a magnetizing current of 8 pu can magnetize the VMMs so as to vary from -100 to $100 \%$ of magnetization for polarity reversal. Under these conditions, the PMs again exhibit reverse polarity; therefore, the proposed motor can change the polarity by magnetizing the VMMs in the rotor.

\subsection{No-load Characteristics in the Reversible Salient} Pole We confirmed a change in the central axis (i.e., the d-axis) of the resultant magnetic flux for both the VMMs and the CMMs under changes to the VMMs' polarity. Magnetic analysis was performed to obtain the no-load characteristics in the model RS-PM motor associated with this polarity reversal. Figure 7 shows the distribution of the magnetic flux 


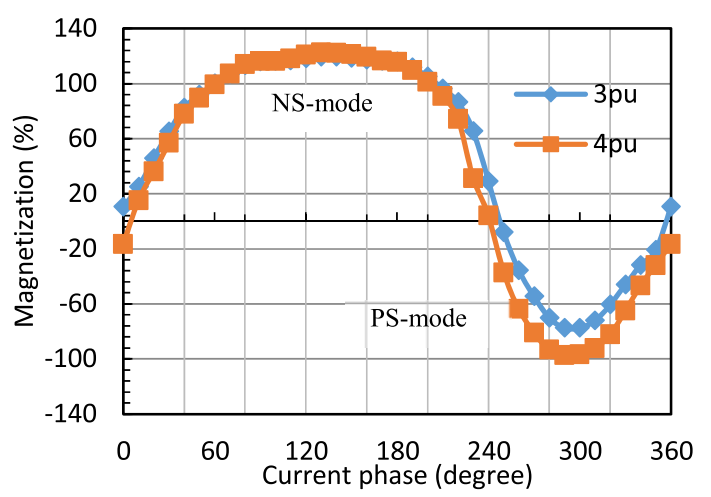

Fig. 3. Magnetization from NS to PS in variable magnetized PM

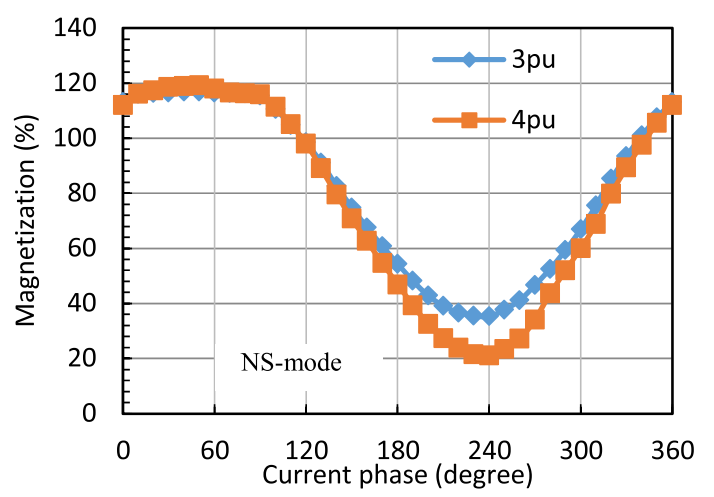

Fig. 4. Magnetization from NS to PS in constant magnetized PM

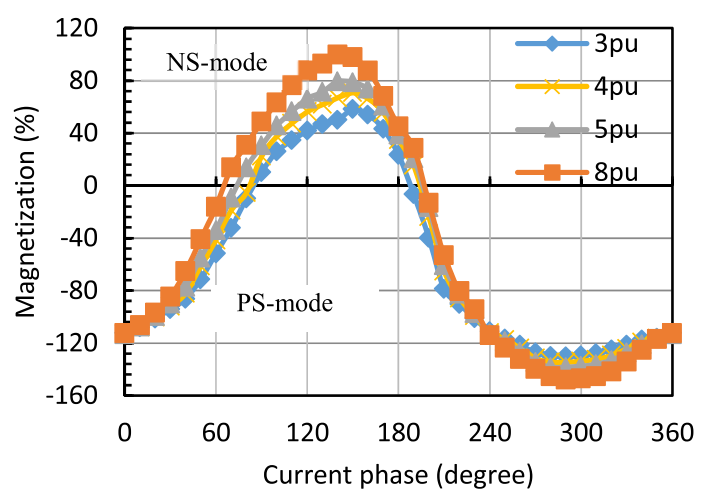

Fig. 5. Magnetization from PS to NS in variable magnetized PM

density due to the change in the polarity of the VMMs under no loading. When a pair of VMM and CMM in the Vshape have the same polarity, the resultant magnetic flux of the PMs forms a magnetic pole in the core of the V-shape and flows through the core between the two V-shapes, as shown in Fig. 7(a). When the a pair of VMM and CMM in the Vshape have opposite polarities, the resultant magnetic flux of PMs forms a magnetic pole in the core between the two Vshapes and the flux flows through the core in the V-shape, as shown in Fig. 7(b). Therefore, the reversible magnetization in the PM enables changes in the salient pole of the PM motor. The flux density of the stator core of the RS-PM is not as high as that of a magnetic-saturated core, such as in an IPM motor designed for a higher magnetic loading. The pole arc of PM becomes smaller than that of a conventional IPM using PMs of maximum volume because the rotor of the

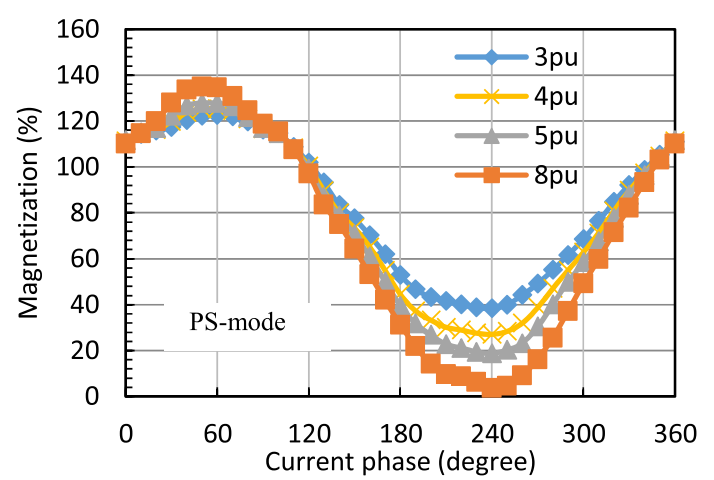

Fig. 6. Magnetization from PS to NS in constant magnetized PM
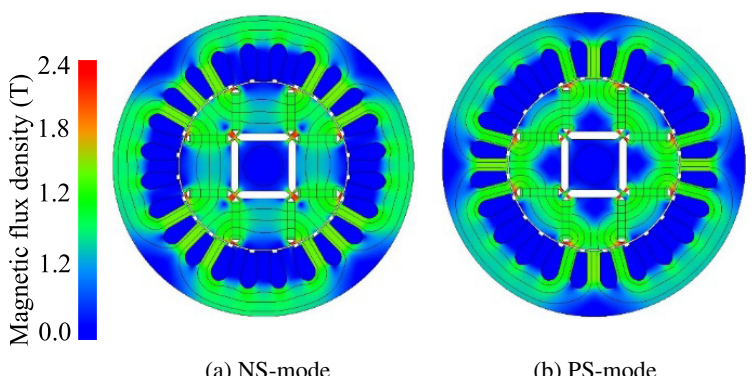

(b) PS-mode

Fig. 7. Distribution of magnetic flux density in the NSand PS-modes

RS-PM has to consist of PM and the core part changing into $\mathrm{d}$-axis or q-axis magnetic-pass. If the RS-PM motor is designed to undergo a magnetic saturation of its core, the salient pole ratio will attain a lower value and the reluctance-torque components will decrease while the torque component due to the PM increases. As a result, the motor will achieve operational characteristics that offer a slightly higher maximum total torque, a narrower range of speeds, and a lower efficiency at high speeds. Figure 8 shows the harmonic components of the back-electromotive force (back-EMF) in the NS- and PSmodes. Each fundamental component of the induced voltage in the two models varies by $13 \%-16 \%$ due to the reversal of the salient pole. When the V-shaped arc is $90^{\circ}$ or $100^{\circ}$, the back-EMFs of the NS- and PS-modes are 151.3 and $131.5 \mathrm{~V}$ at $90^{\circ}$ and 145.5 and $122.8 \mathrm{~V}$ at $100^{\circ}$, respectively. The differences between the back-EMFs of the NS- and PS-modes are $13 \%$ and $16 \%$, respectively, as shown in Fig. 8 . This result implies that the motor in the case of the PS-mode should operate over a wider range of speeds with a smaller negative $\mathrm{d}$-axis current for flux-weakening control at high speed because of a smaller back-EMF, whereas the maximum torque will decrease due to the slightly smaller flux linkage of the PM.

3.4 Salient Ratio of d-axis and q-axis Inductance

The inductance of the d-q-axis is an important factor because it determines the characteristics of IPM motors, particularly the motor's performance under variable-speed operation. In addition, the salient ratio and characteristics are determined from the motor's d- and q-axis inductances ( $\mathrm{Ld}$ and Lq, respectively). Obtaining the salient ratio of the machine is important since it is the main factor in determining the reluctance-torque component and the current-phase at peak torque. Each inductance was derived from magnetic-field 


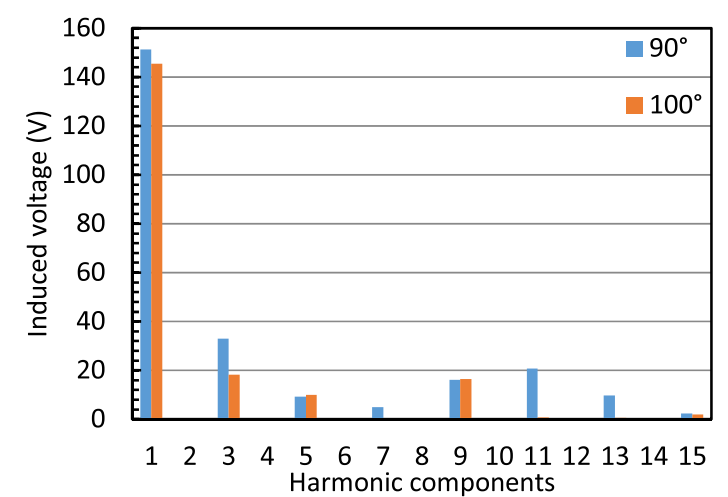

(a) NS-mode

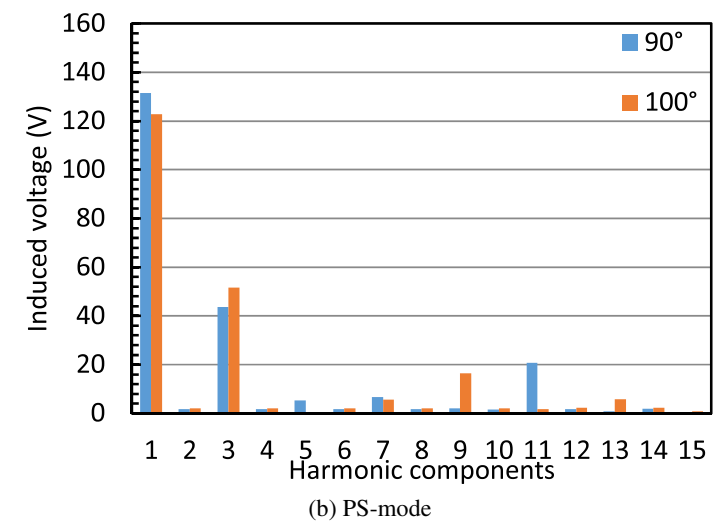

Fig. 8. Distribution of magnetic flux density in the NSand PS-modes

analysis. $\mathrm{Ld}, \mathrm{Lq}$, and the salient ratio $(\mathrm{Lq} / \mathrm{Ld})$ are shown in Fig. 9. While the Ld of the NS-mode (negative saliency) decreases with an increasing V-shaped-arc angle, that of the PSmode (positive saliency) increases. Furthermore, the Ld of the PS-mode is higher than that of the NS-mode because the PS-mode has a wide d-axis magnetic pass between neighboring PMs. Furthermore, the Ld increases with the V-shapedarc angle because the sectional area of the $\mathrm{d}$-axis magnetic pass is wider. While the Lq of the NS-mode increases with increasing arc angle, that of the PS-mode decreases. As a result, the salient ratio of the NS-mode increases from 1.35 to 1.64 , implying that the larger V-shaped arc of $100^{\circ}$ provides a higher torque due to the increased reluctance torque component in the negative salient pole. The salient ratio of the PS-mode decreases from 0.82 to 0.64 when the angle of the V-shaped arc changes from $90^{\circ}$ to $100^{\circ}$, implying that the larger V-shaped arc of $100^{\circ}$ provides a higher torque because of the increased reluctance-torque component in the positive salient pole.

3.5 Relationship between Torque and Phase of Current The phase of the current at which the PM motor produces its maximum torque varies depending on the salient ratio of the poles in the machine. Magnetic field analysis was performed on two models of the RS-PM motor to obtain the dependence of torque characteristics upon the current phase. The phase of the current was set by the spatial orientation of the stator magnetomotive force (MMF) with respect to the d-axis for the two modes. To do so, torque was plotted as a function of the phase based on FEM analysis. Figures 10(a) and 10(b) show the torque-phase current characteristics of the NS- and PS-modes, respectively. Each
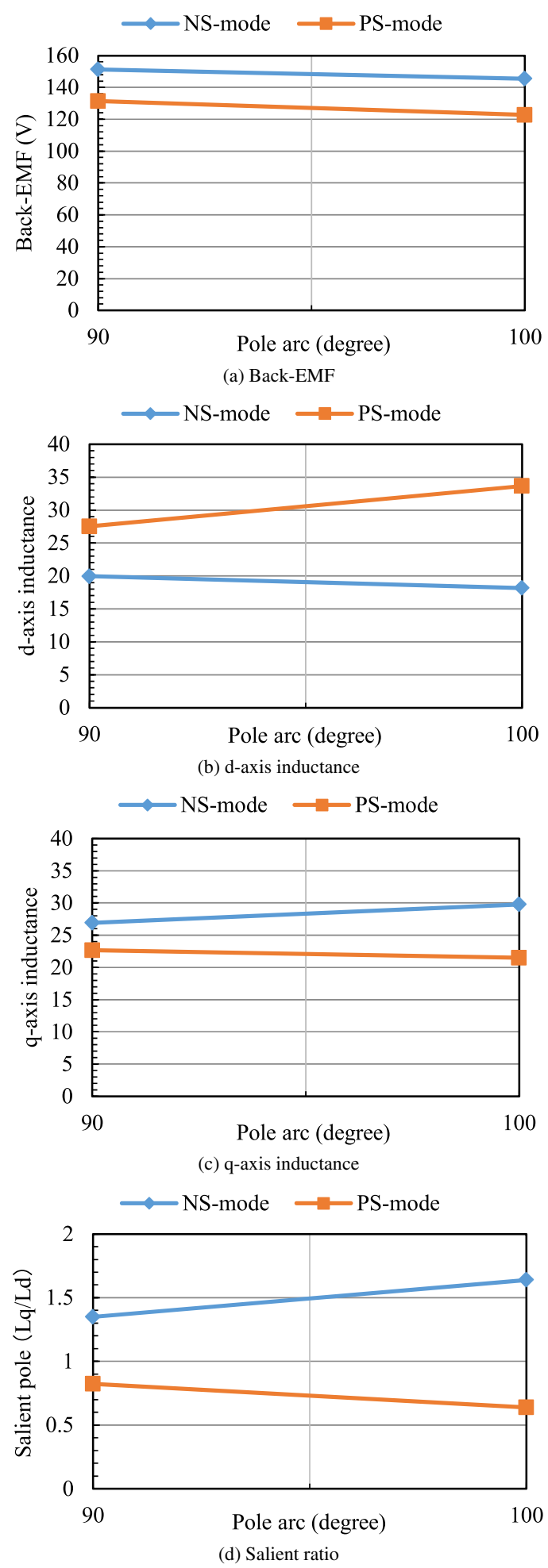

Fig. 9. Motor constants vs. V-shaped arc in NS- and PSmodes

current is at a rated value of $5 \mathrm{~A}$. When the V-shaped-arc angle is $90^{\circ}$, the NS-mode is a negative salient pole since the phase at the maximum torque is $103^{\circ}$. The PS-mode is also a positive salient pole when the $\mathrm{V}$-shaped arc is $90^{\circ}$ because the phase at the maximum torque is $75^{\circ}$. When the V-shapedarc angle is $100^{\circ}$, the NS-mode is a negative salient pole because the phase at the maximum torque is $110^{\circ}$ (which is larger than $90^{\circ}$ ), whereas the PS-mode is a positive salient 


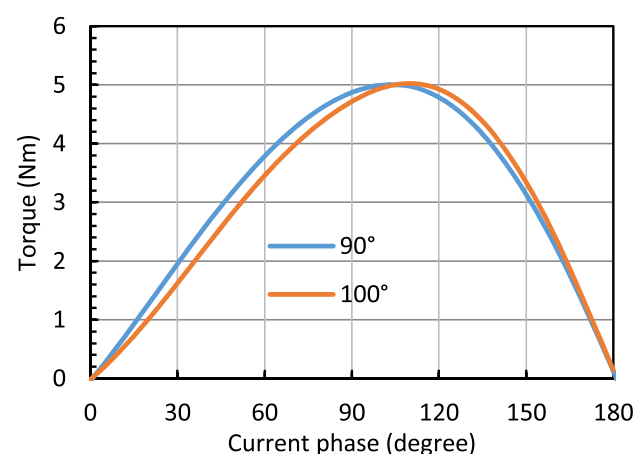

(a) NS-mode

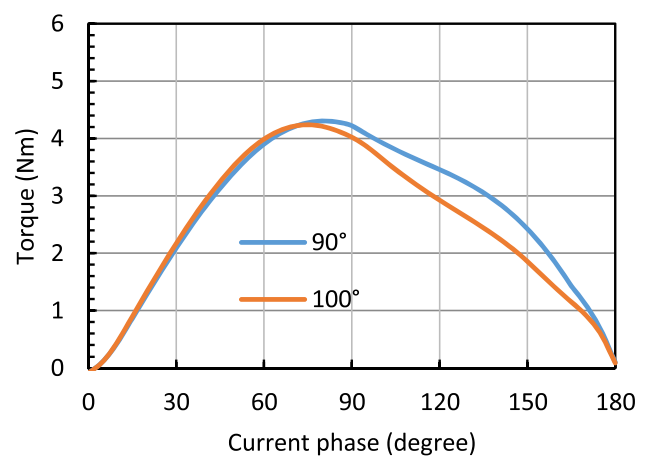

(b) PS-mode

Fig. 10. Torque characteristics of the current phase in the NS- and PS-modes for each V-shaped arc

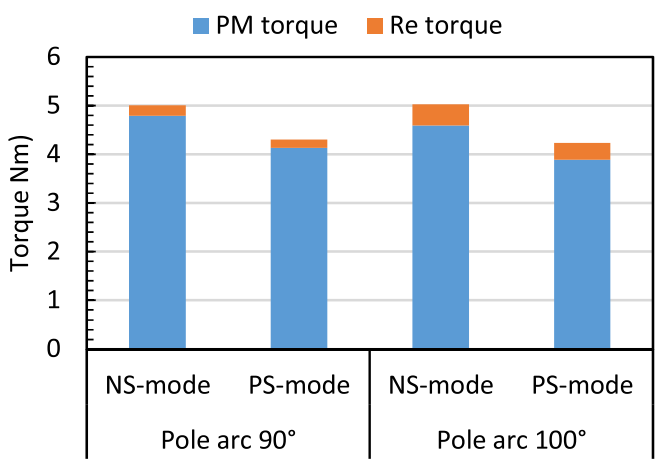

Fig. 11. Torque components in the NS- and PS-modes for each V-shaped arc

pole because the phase current at the maximum torque is $75^{\circ}$, which is smaller than $90^{\circ}$. After evaluating the reluctance torque components of the total torque and conducting magnetic field analysis under the assumption that all PMs in the motor are not magnetized, we found that the curve of the reluctance-torque components was symmetric about a phase of $90^{\circ}$ and that the reluctance torque curves in the NS- and PS-modes had opposite polarities. Therefore, the direction of saliency was reversed between the NS-and PS-modes, and the V-shaped-arc angle influenced the saliency of the RS-PM motor.

3.6 Torque Components The RS-PM motor is a type of IPM motor, owing to the fact that it exhibits a variation in reluctance due to the magnetic rotor core. IPMs produce a motor torque that combines magnetic torque with reluctance torque, allowing us to understand the full set of torque components. In order to produce only the reluctance torque component, magnetic field analysis was performed

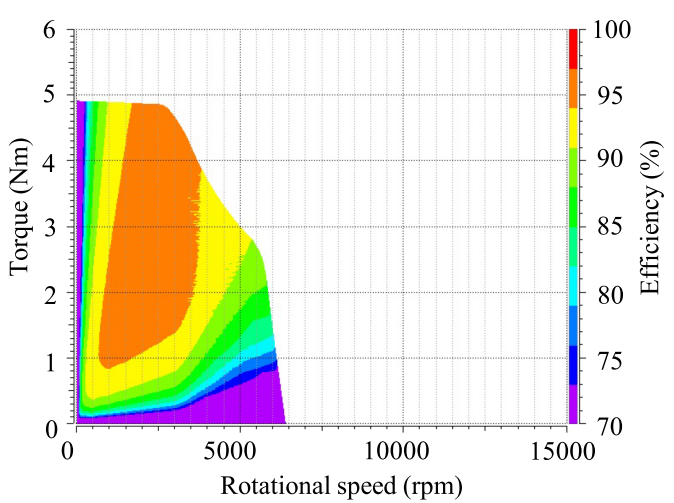

(a) NS-mode

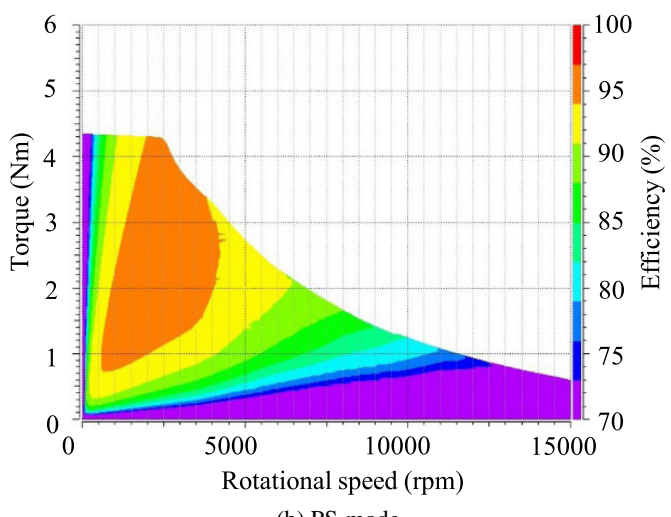

Fig. 12. Efficiency over a wide range of speeds for $90^{\circ}$ V-shaped arc

assuming that no PMs in the motor are magnetized. Figure 11 shows the torque components in each V-shaped arc $\left(90^{\circ}\right.$ and $100^{\circ}$ ). When the V-shaped arc is $90^{\circ}$, the NS-mode PM torque is $4.78 \mathrm{Nm}$, and the corresponding reluctance torque is $0.22 \mathrm{Nm}$; in the PS-mode, the PM torque is $4.13 \mathrm{Nm}$, and the reluctance torque is $0.17 \mathrm{Nm}$. When the $\mathrm{V}$-shaped arc is $100^{\circ}$, the PM and reluctance torques in the NS-mode are $4.59 \mathrm{Nm}$ and $0.43 \mathrm{Nm}$, respectively; in the PS-mode, the PM torque is $4.24 \mathrm{Nm}$, and the reluctance torque is $0.35 \mathrm{Nm}$. These results confirm that, in both the NS- and PS-modes, the ratio of reluctance torque to total torque increases as the angle of the $\mathrm{V}$-shaped arc increases.

3.7 Variable-speed Motor Performance In variablespeed drive systems, such as electric vehicles (EVs) and wind generators, a wider range of speeds and a higher efficiency improve the performance of motor-drive systems. We discuss motor performance at variable speeds in order to operate over a wider speed range. The motor's torque, voltage, and efficiency vary based on the angle of the V-shaped arc and the mode of the motor (i.e., the NS-mode or PS-mode). The relationship between torque and speed characteristics was determined from our FEM-based magnetic-field analysis. Figures 12 and 13 show the motor performances for different speeds and V-shaped arc angles. In the NS-mode, the maximum torque of each $\mathrm{V}$-shaped arc is almost the same: $5.0 \mathrm{Nm}$. The base speed under each V-shaped arc is 2,700 rpm, and the top speed ranges from 6,250 to $6,400 \mathrm{rpm}$. In the PS-mode, the maximum torque under each $\mathrm{V}$-shaped arc is almost the same: $4.2-4.3 \mathrm{Nm}$. The $90^{\circ} \mathrm{V}$-shaped arc has a slightly larger maximum torque than the $100^{\circ} \mathrm{V}$-shaped arc. The base speed 


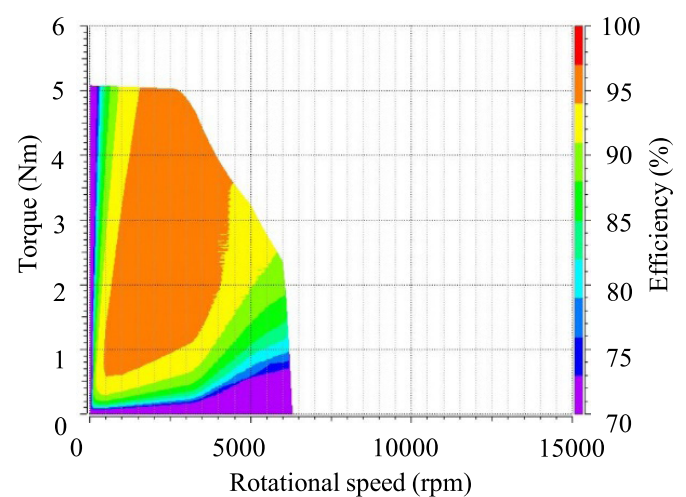

(a) NS-mode

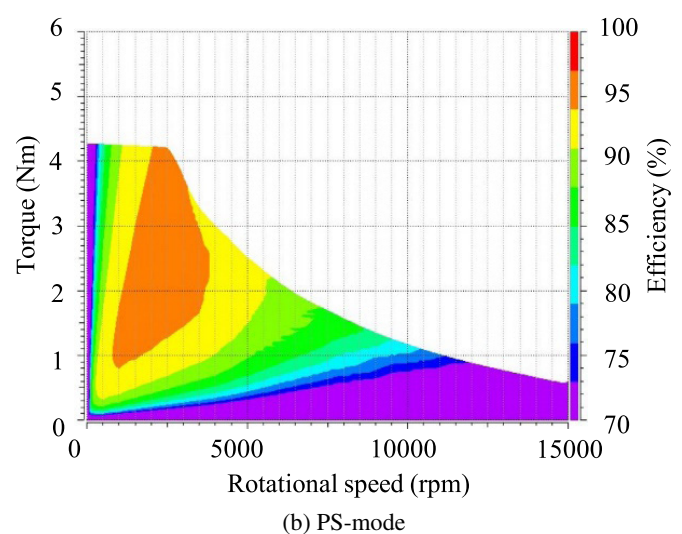

Fig. 13. Efficiency over a wide range of speeds for $100^{\circ}$ V-shaped arc

of each V-shaped arc is 2,500 rpm, and the top speed is beyond $15,000 \mathrm{rpm}$. The output power, which is obtained as the product of torque and speed, is larger for the $90^{\circ} \mathrm{V}$-shaped arc model than for the $100^{\circ}$ model. These results indicate that the maximum torque in the NS-mode is larger than that in the PS-mode at low speeds for both V-shaped-arc angles. Furthermore, the top speed in the PS-mode is more than twice that in the NS-mode, and the torque in the PS-mode is larger than that in the NS-mode at speeds exceeding 5,600 rpm for both V-shaped-arc angles. Thus, the PS-mode enhances the output power at high speeds, and each mode (due to the reversible salient pole) enhances the torque at low speeds and the output power at high speeds.

3.8 Efficiency over a Wide Range of Speeds The high efficiency of the motor over the entire operational range results in lower energy consumption because the variablespeed motor operates from low speed to high speed and from low power to high power. Motor efficiency is strongly dependent on rotational speed and torque, which means that machine constants (such as d-axis and q-axis inductance and flux linkage due to a PM field) are dominant factors in determining motor performance. Figures 12 and 13 show the efficiency distributions for different speeds in the NS- and PS-modes for each V-shaped-arc angle. When evaluating the overall efficiency over a wide range of speeds and torques, we find that the motors with $90^{\circ}$ and $100^{\circ} \mathrm{V}$-shaped arcs achieve high levels of efficiency. In the NS-mode, the motor with the $100^{\circ} \mathrm{V}$-shaped arc can operate at its highest efficiency over a wider range of speeds than the motor with the $90^{\circ}$ $\mathrm{V}$-shaped arc. On the other hand, in the PS-mode, this situ-

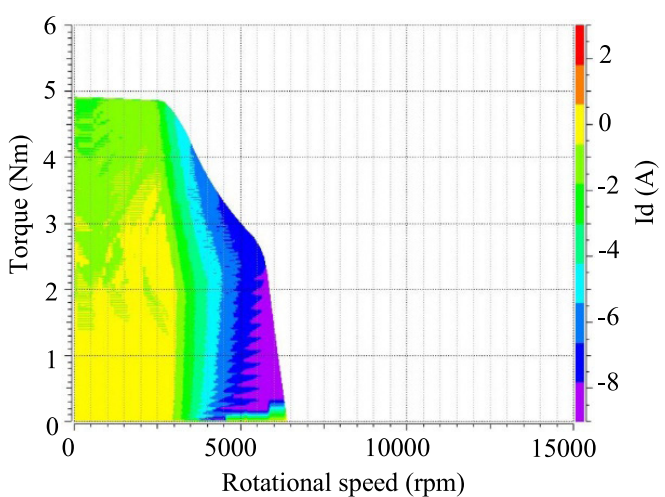

(a) NS-mode

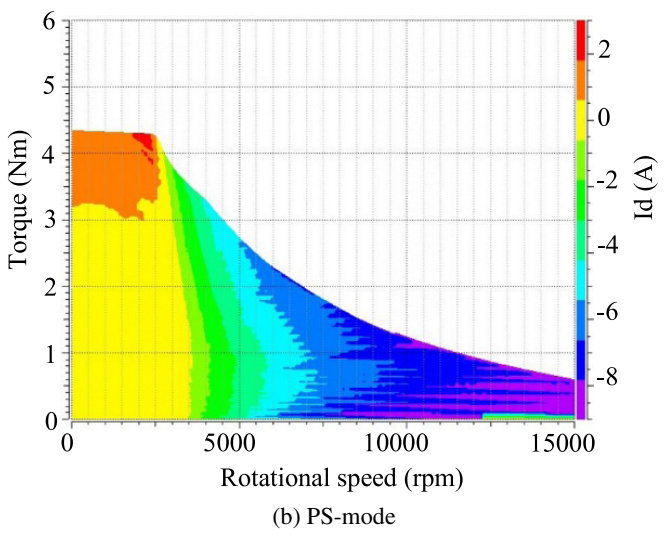

Fig. 14. d-axis current for $90^{\circ} \mathrm{V}$-shaped arc

ation is reversed. Overall, the efficiency at high speed in the PS-mode is higher than that in the NS-mode. Therefore, RSPM motors that combine operations in the NS-mode at low speeds and the PS-mode at high speeds provide high power and efficiency over a wide range of speeds.

Figures 14 and 15 show the d-axis and q-axis currents for the efficiency distributions in the NS- and PS-modes shown in Figs. 12 and 13. At medium- and high-rotational speeds, the d-axis currents in both the NS- and PS-modes increase along with the rotational speed since the use of flux-weakening control causes the motor's voltage to remain less than the upper limit of the source voltage. Flux-weakening control is performed by shifting the current phase, thereby increasing the negative d-axis current component. The d-axis current maps indicate that the negative $\mathrm{d}$-axis current increases as the rotational speed increases beyond the base rotational speed. Furthermore, the negative d-axis current in the PS-mode is smaller than that in the NS-mode because the d-axis inductance is larger in the PS-mode due to that mode's wider magnetic pass of the outer periphery between neighboring PMs. Another reason for this difference is the smaller flux linkage in the PS-mode due to its PMs. Therefore, the negative d-axis current in the PS-mode is smaller than that in the NS-mode. At low speeds (i.e., up to the base value), the NS-mode operates near the phase current at which the motor produces its maximum torque. This is also the case for the PS-mode. The positive d-axis current increases with torque up to the base rotational speed in the PS-mode. The total torque in the NS-mode is larger than that in the PS-mode because each flux linkage of the PMs in the NS-mode is larger than that in the PS-mode, and each reluctance torque component is larger 


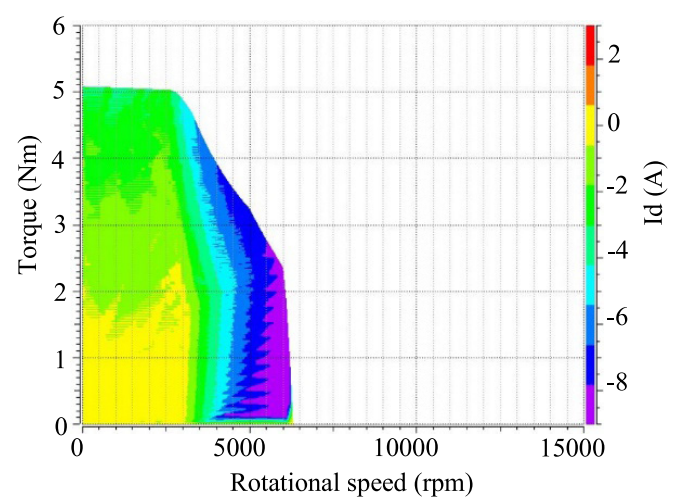

(a) NS-mode

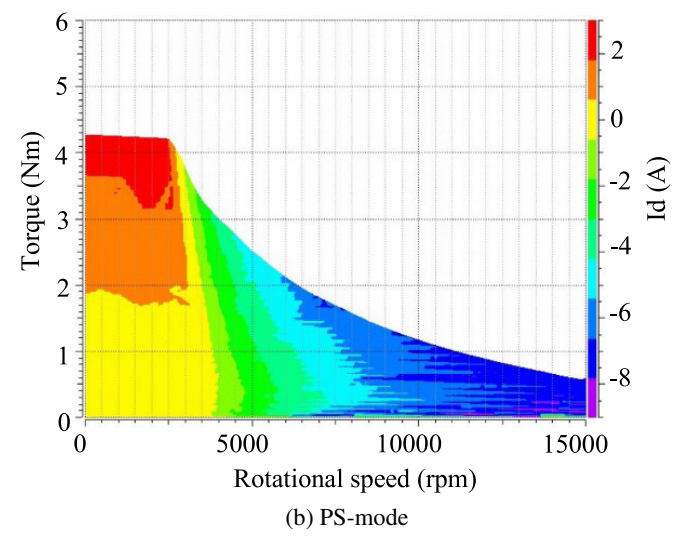

Fig. 15. d-axis current for $100^{\circ} \mathrm{V}$-shaped arc

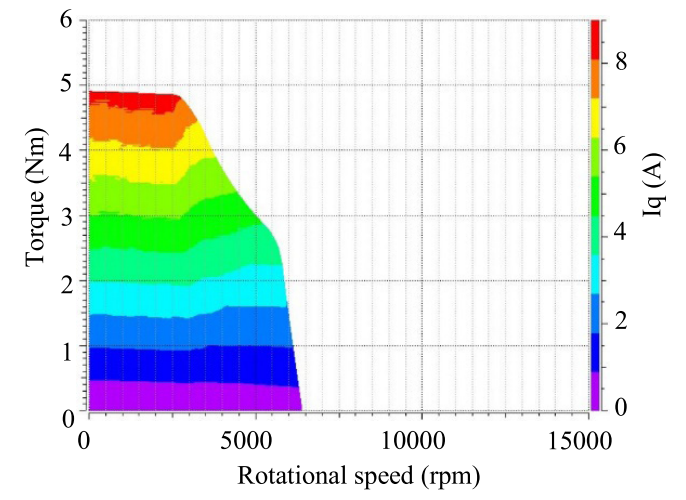

(a) NS-mode

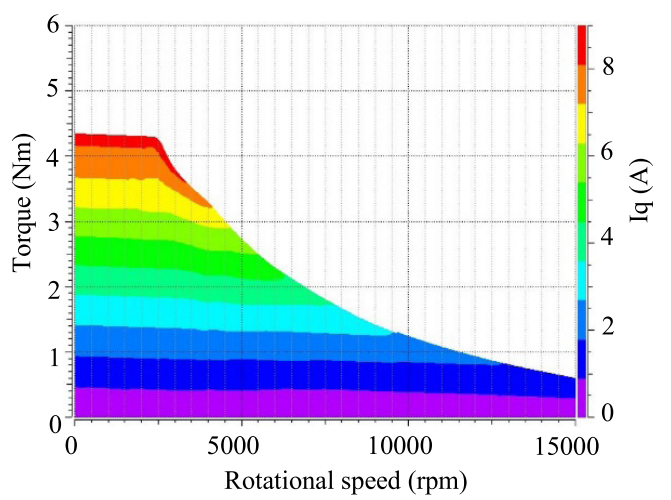

(b) PS-mode

Fig. 16. q-axis current for $90^{\circ} \mathrm{V}$-shaped arc

than that in the PS-mode.

3.9 Loss over a Wide Range of Speeds Figures 18 and 19 show the iron-loss and copper-loss distributions,

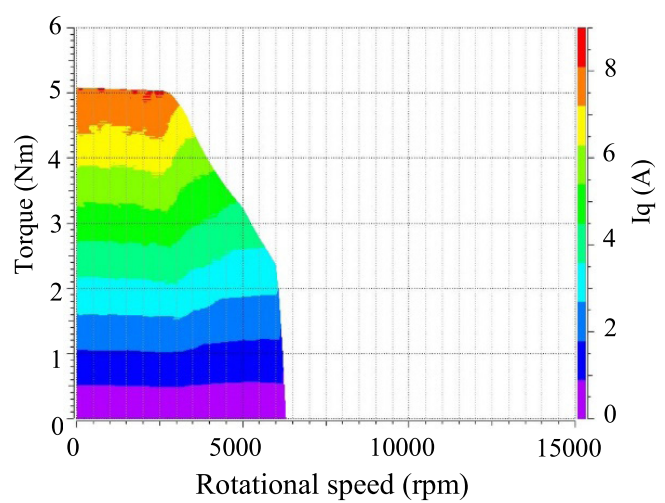

(a) NS-mode

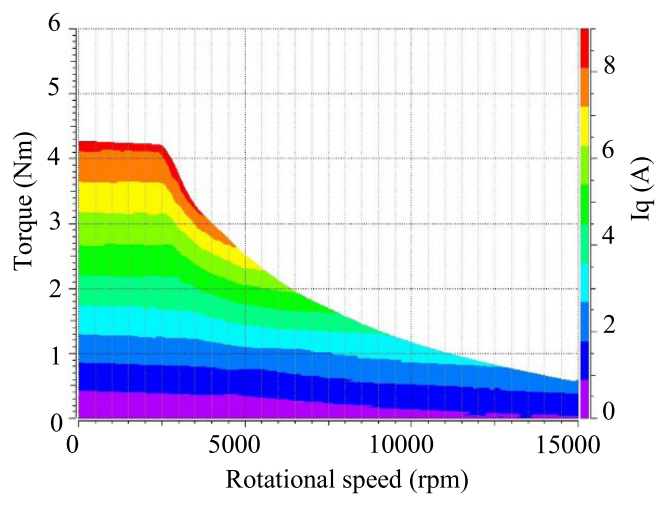

(b) PS-mode

Fig. 17. q-axis current for $100^{\circ} \mathrm{V}$-shaped arc

respectively, at different speeds for the NS- and PS-modes. The iron loss in the NS-mode increases with increasing rotational speed because the frequency increases, as shown in Fig. 18(a). In particular, the iron loss increases drastically at speeds beyond the base speed $(2,500 \mathrm{rpm})$. The copper loss increases with torque because of a corresponding increase in Iq, as shown in Figs. 18(b) and 16(a). Furthermore, copper loss dramatically increases at speeds beyond the base speed (from 2,500 to 6,500 rpm) because Id substantially increases to suppress the overvoltage due to the use of the flux-weakening control. Similarly, the iron loss in the PS-mode increases with rotational speed in the region above about 5,000 rpm, as shown in Fig. 19(a). In the PS-mode, the copper loss increases with torque because of a corresponding increase in Iq, as shown in Figs. 19(b) and 16(b). Furthermore, copper loss increases at speeds exceeding the base speed (from 2,500 to $15,000 \mathrm{rpm}$ ) because Id substantially increases to suppress the overvoltage when the flux-weakening control is employed.

\section{Motor Performance Due to Variable Magnetic Force}

We now discuss the performance of the RS-PM motor with reversible saliency and variable magnetic force in the $\mathrm{PM}^{(5)(7)}$. A negative magnetizing current demagnetized the VMMs in each reversible salient mode. We analyzed the characteristics of the RS-PM motor for residual magnetic flux densities (Br) of $90 \%, 80 \%$, and $70 \%$ in variable magnetized PMs. Next, we discuss the performance of the motor at different drive speeds. The torque-versus-speed characteristics were obtained from FEM-based magnetic-field analyses. 


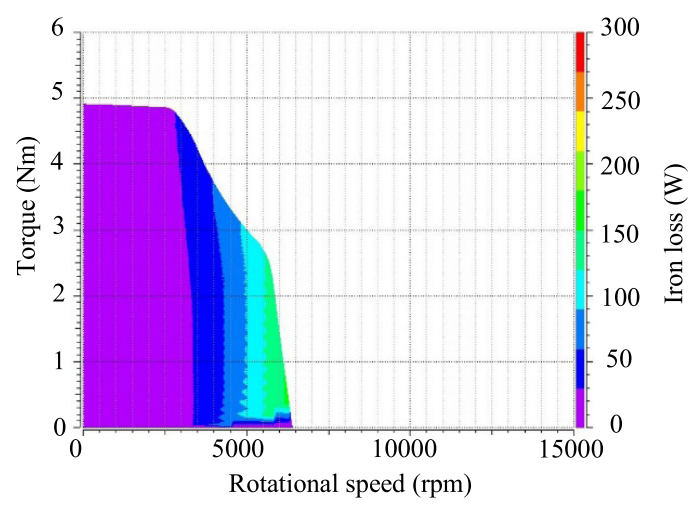

(a) Iron loss in the NS-mode

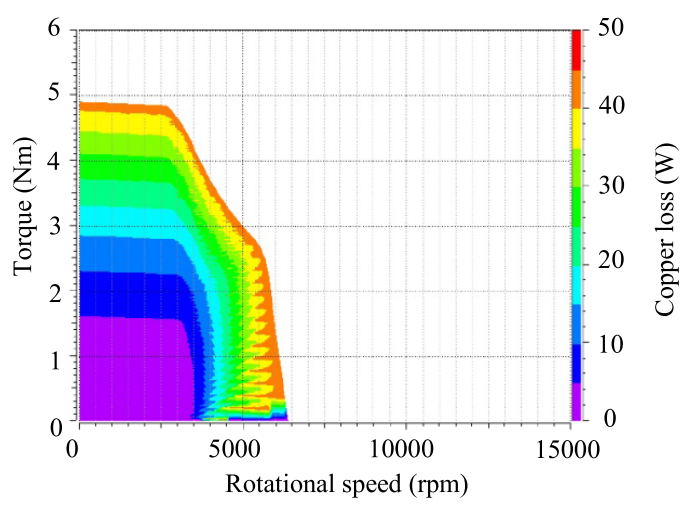

(b) Copper loss in the NS-mode

Fig. 18. Iron loss and copper loss in the NS-mode for $90^{\circ} \mathrm{V}$-shaped arc

The maximum voltage was $250 \mathrm{~V}$ (177 Vrms), and the effective maximum current was 5 Arms. Figure 20(a) shows the characteristics of the motor operating in the NS-mode when the VMMs are demagnetized from $100 \%$ to $70 \% \mathrm{Br}$ by the magnetizing current. The results indicate that the maximum speed in the case of $70 \% \mathrm{Br}$ is approximately $10,000 \mathrm{rpm}$, which is about 1.6-times that in the case of $100 \% \mathrm{Br}$. The maximum power (equal to the torque times the rotational speed) is larger than that in the case of $100 \% \mathrm{Br}$ at high rotational speeds. Figure 20(b) shows the characteristics of the motor operating in the PS-mode when VMMs are demagnetized from $100 \%$ to $70 \% \mathrm{Br}$ by the magnetizing current. The results indicate that the maximum speed at $70 \% \mathrm{Br}$ is over 15,000 rpm; however, the maximum power (equal to torque times rotational speed) is smaller than that in the case of $100 \% \mathrm{Br}$ under all rotational speeds.

Next, we discuss the efficiency of RS-PM at different drive speeds when the VMMs are demagnetized to $90 \%, 80 \%$, and $70 \% \mathrm{Br}$ by the demagnetizing current (as above). Figures 21 and 22 show the efficiency distributions at different speeds and torques when the VMMs are demagnetized from $100 \%$ to $70 \%$ of $\mathrm{Br}$ in the NS- and PS-modes, respectively.

In the NS-mode, the efficiencies in the cases of demagnetization to $90 \%, 80 \%$, and $70 \% \mathrm{Br}$ for torques of less than about $3 \mathrm{Nm}$ were found to be higher than those in the nondemagnetized case (100\% of Br). In the PS-mode, these efficiencies for torques less than about $1 \mathrm{Nm}$ were also higher than that in those non-demagnetized case $(100 \% \mathrm{Br})$. Therefore, the combination of a reversible salient pole with a variable magnetic PM force provides high efficiency over a wide

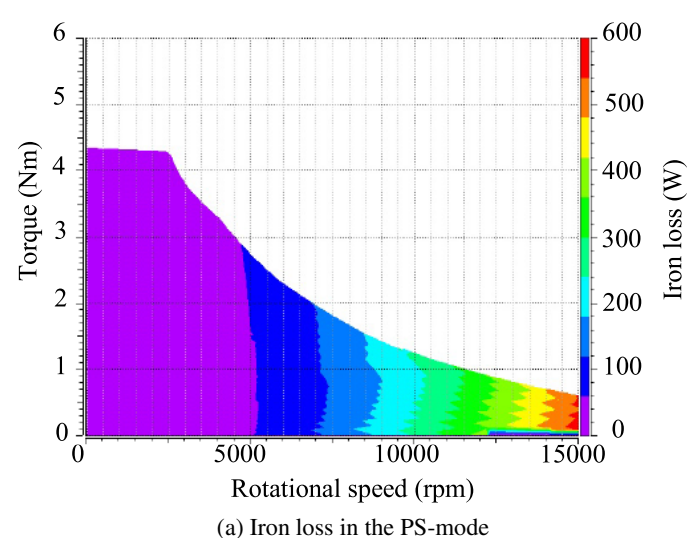

(a) Iron loss in the PS-mode

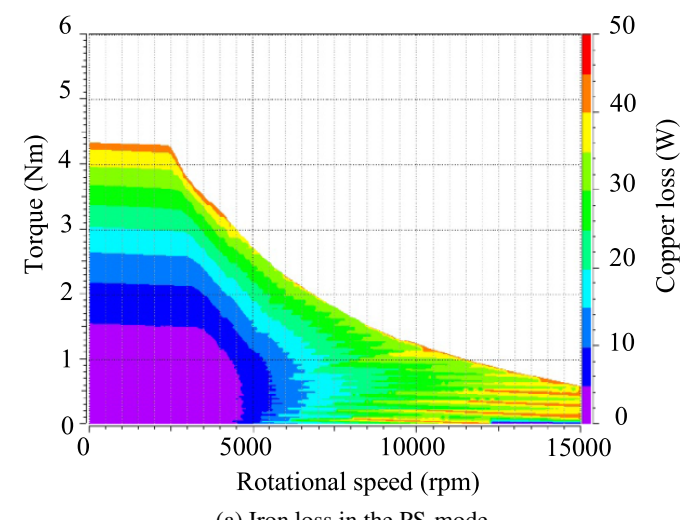

Fig. 19. Iron loss and copper loss in the PS-mode for $90^{\circ}$ V-shaped arc

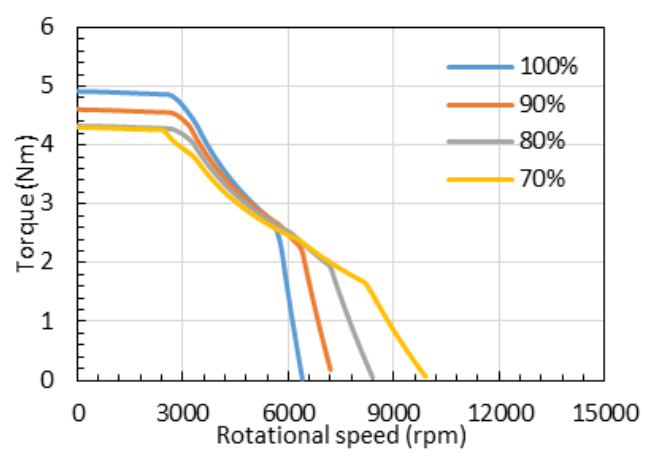

(a) NS-mode

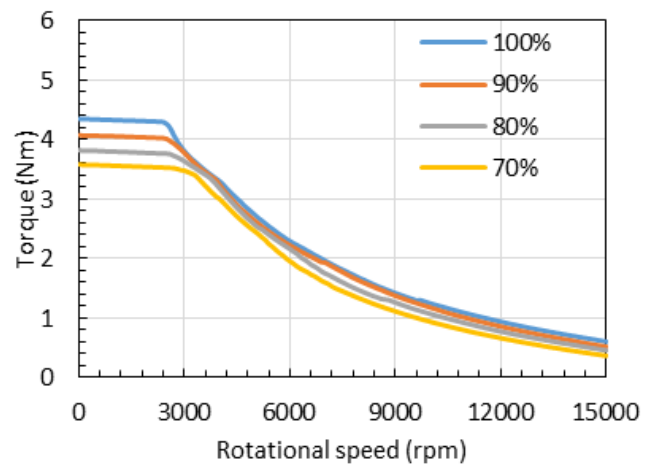

(b) PS-mode

Fig. 20. Motor performance over a wide range of speeds for a $90^{\circ} \mathrm{V}$-shaped arc for variable magnetic force

range of speeds and torques for variable-speed driving. 


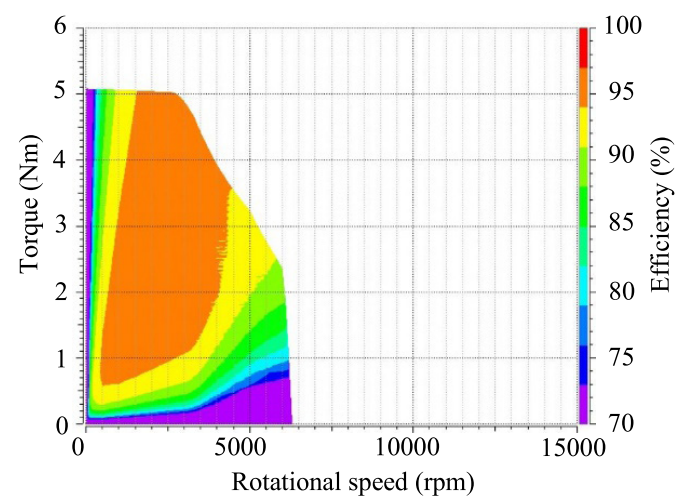

(a) $\mathrm{Br}=100 \%$

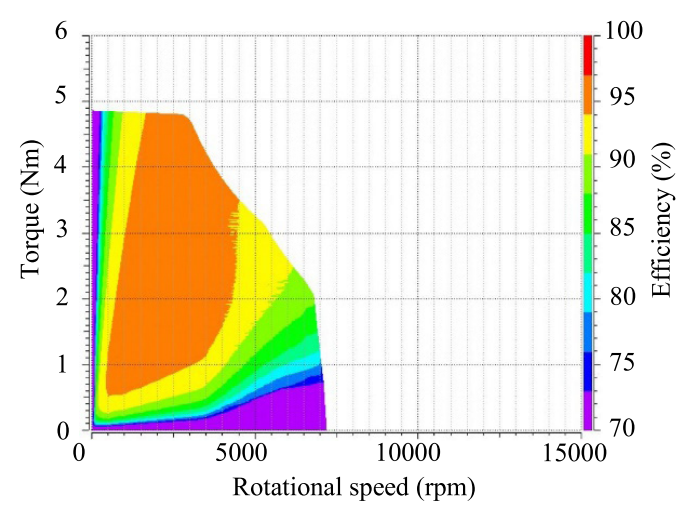

(b) $\mathrm{Br}=90 \%$

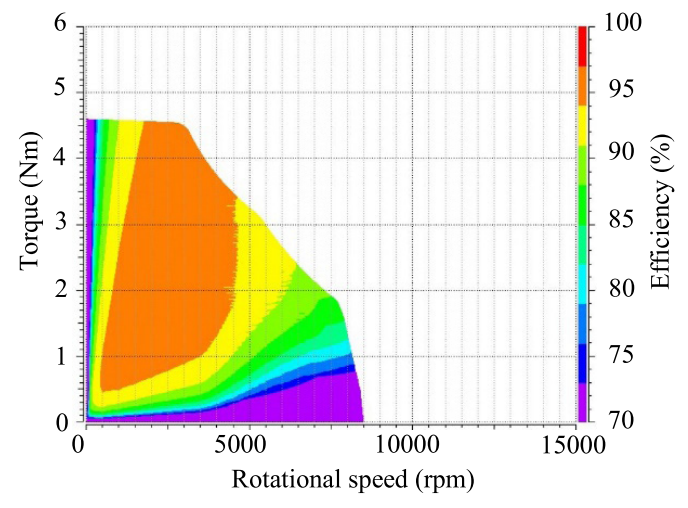

(c) $\mathrm{Br}=80 \%$

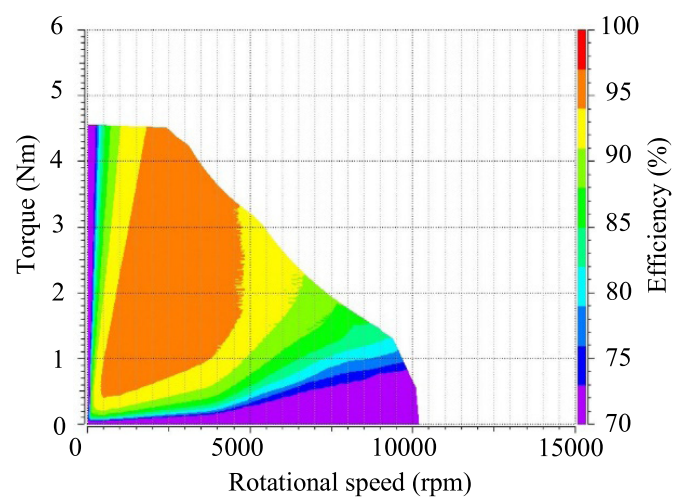

(d) $\mathrm{Br}=70 \%$

Fig. 21. Efficiency in the NS-mode over a wide range of speeds for a $90^{\circ} \mathrm{V}$-shaped arc for variable magnetic force (demagnetization)

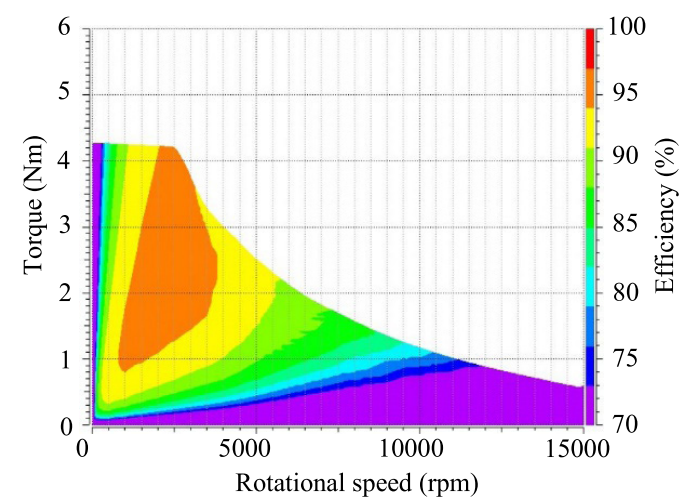

(a) $\mathrm{Br}=100 \%$

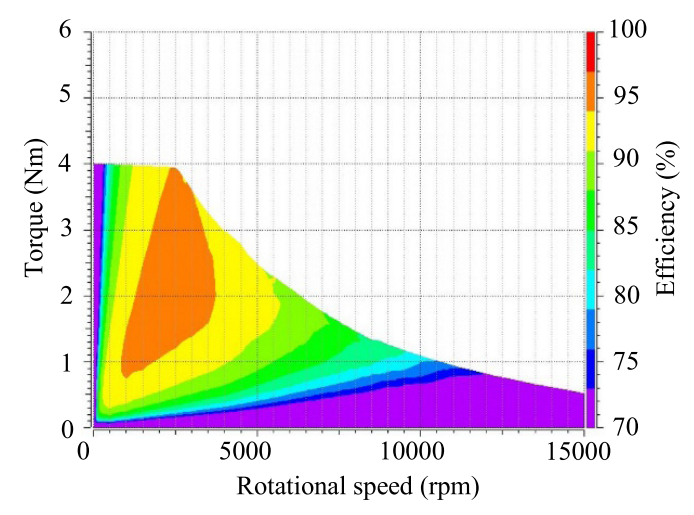

(b) $\mathrm{Br}=90 \%$

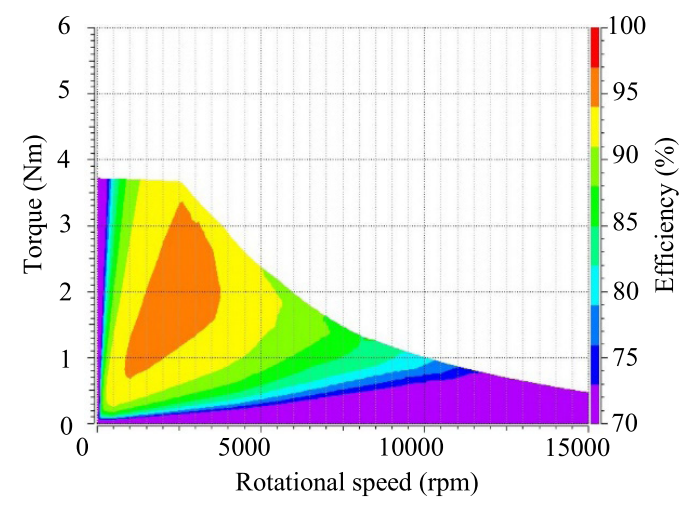

(c) $\mathrm{Br}=80 \%$

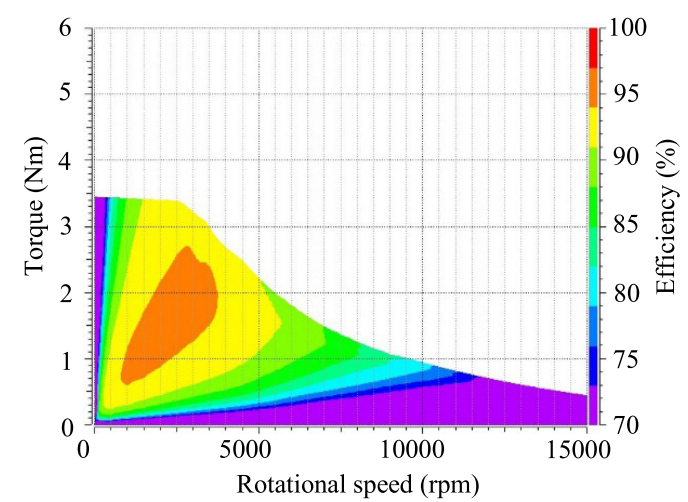

(d) $\mathrm{Br}=70 \%$

Fig. 22. Efficiency in the PS-mode over a wide range of speeds for a $90^{\circ} \mathrm{V}$-shaped arc for variable magnetic force (demagnetization) 


\section{Conclusion}

This study proposed a novel PM motor with reversible salient poles. The rotor configuration and working principles of the motor were discussed, and the characteristics resulting from the reversible saliency and magnetization were examined. The results of our analysis confirm that the proposed motor (RS-PM) can alternate its pole's direction of saliency from negative to positive through the magnetization of the PM. The motor's performance indicates that the reversible salient pole allows the motor to achieve high torque at low speeds, high power at high speeds, and higher efficiency over a wide range of speeds. Furthermore, the combination of reversible saliency with variable magnetic force in the PM allows the motor to attain high efficiency at a low output power.

Therefore, the proposed motor can be utilized to deliver high performance and efficiency in a variable-speed drive system.

\section{References}

( 1 ) A. Weschta: "Schwächung des Erregerfeldes bei einer dauermagneterregren Synchronmaschine", TZ Arcb., Vol.7, pp.79-84 (1985)

( 2 ) L.R. Herman: The Roesel Generator-Constant frequency with variable speed", Proc. 1976 IEEE Power Engineering Society Winter Meeting, paper A, pp.76035-76036 (1976)

( 3 ) V. Ostovic: "Memory Motors", Ind. Appl. Mag., Vol.9, pp.52-61 (2003)

( 4 ) V. Ostovic: "Pole-changing permanent-magnet machines", IEEE Trans. Ind. Appl., Vol.38, pp.1493-1499 (2002)

( 5 ) K. Sakai, K. Yuki, Y. Hashiba, N. Takahashi, and K. Yasui: "Principle of the variable-magnetic-force memory motor", ICEMS, LS6A-1 (2009)

( 6 ) K. Sakai, D. Misu, K. Yuki, K. Yasui, Y. Hashiba, and N. Takahashi: "New generation motor for energy saving", Proc. 2010 International Power Electronics Conf., pp.1354-1358 (2010)

( 7 ) K. Sakai, K. Yuki, Y. Hashiba, N. Takahashi, K. Yasui, and L. Kovudhikulrungsri: "Principle and basic characteristics of variable-magnetic-force memory motors", IEEJ Trans. IA, Vol.131, pp.53-60 (2011) (in Japanese)
( 8 ) K. Sakai and N. Yuzawa: "Permanent magnet motors capable of pole changing and tree-torque-production mode using magnetization", IEEJ Trans. Ind. Appl., Vol.2, pp.269-275 (2013)

( 9 ) K. Sakai and N. Yuzawa: "Permanent magnet motor capable of pole changing for high efficiency", Proc. 2013 IEEE ECCE, pp.5064-5071 (2013)

(10) T. Kato, N. Limsuwan, Chen-Yen, K. Akatsu, and R.D. Lorenz: "Rare earth reduction using a novel variable magnetomotive force intensified IPM machine", IEEE Trans. Ind. Appl., Vol.50 pp.1748-1756 (2014)

(11) K. Matsuda and K. Sakai: "A PM motor capable of conversion for salientpole direction", 2015 National Convention Record, IEE Japan, pp.5-18 (2015) (in Japanese)

(12) K. Matsuda and K. Sakai: "A permanent magnet motor with reversible salient poles", Proc. 2015 JIAS Conf., pp.3-33 (2015) (in Japanese)

(13) K. Sakai and K. Matuda, "A permanent magnet motor with reversible salient poles”, in Proc. 2015 IEEE ECCE, pp.1757-1764 (2015)

Kazuto Sakai (Senior Member) received a Dr. Eng. degree from

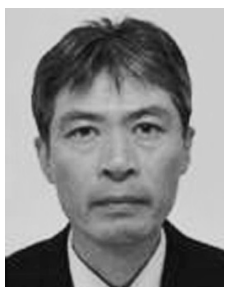
Yamaguchi University, Japan. He worked at Toshiba Corporation, Power and Industrial Systems Research and Development Center from 1983 to 2010, where he developed an AC motor drive technology for servomotors, railroad and hybrid vehicles, and superhigh-speed generators for cogeneration. He joined Toyo University in 2010 as a Professor in the department of Electrical, Electronic and Communication Engineering. His technical efforts have included the research of energy conversion and power electronics; high-performance PM motors and induction motor drives for electric vehicles and railways, and wireless power transfer using electromagnetic resonant coupling.

Kouki Matsuda (Student Member) received a B.Eng. degree from

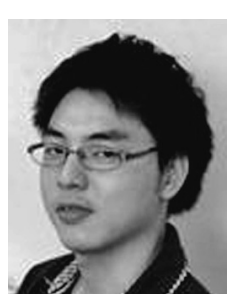
Toyo University, Japan. He is enrolled in the course of Electricity, Electronics and Communications, graduate school of Science and Engineering, Toyo University as of 2014. His technical efforts include the research of high-performance PM machines for electric vehicles and energy conversion systems. 